\title{
miR-27a-3p negatively regulates osteogenic differentiation of MC3T3-E1 preosteoblasts by targeting osterix
}

\author{
YUEXIN XU ${ }^{1-3 *}$, DONG LI $^{4 *}$, ZHU ZHU ${ }^{1}$, LINGYUN LI ${ }^{2}$, YUCUI JIN ${ }^{2}$, CHANGYAN MA $^{2}$ and WEI ZHANG ${ }^{1}$ \\ ${ }^{1}$ Research Institute of Stomatology, Nanjing Medical University, Stomatological Hospital of Jiangsu Province, Nanjing, \\ Jiangsu 210029; ${ }^{2}$ Department of Medical Genetics, Nanjing Medical University, Nanjing, Jiangsu 211166; \\ ${ }^{3}$ Department of Gynecology and Obstetrics, Northern Jiangsu People's Hospital, Yangzhou, Jiangsu 225001; \\ ${ }^{4}$ Department of Orthopedics, Jiangsu Province Hospital of TCM, Affiliated Hospital of Nanjing University \\ of Chinese Medicine, Nanjing, Jiangsu 210029, P.R. China
}

Received September 23, 2019; Accepted April 4, 2020

DOI: $10.3892 / \mathrm{mmr} .2020 .11246$

\begin{abstract}
Osteoporosis is a complex multifactorial disorder characterized by microarchitectural deterioration, low bone mass, and increased risk of fractures or broken bones. Balanced bone remodeling is tightly regulated by the differentiation, activity and apoptosis of bone-forming osteoblasts and bone-resorbing osteoclasts. MicroRNAs (miRs) are dysregulated in osteoporosis, but whether they control osteogenic differentiation and skeletal biology, or could serve as therapeutic targets remains to be elucidated. The present study identified miR-27a-3p as a critical suppressor of osteoblastogenesis. Bioinformatics analysis and luciferase reporter assays demonstrated that miR-27a-3p directly targeted and controlled the expression of osterix (Osx), an early response gene essential for bone formation, through its 3 '-untranslated region. miR-27a-3p functionally inhibited the differentiation of preosteoblasts by decreasing Osx expression, which synergistically contributed to bone formation. miR-27a-3p level was significantly decreased during osteogenic differentiation and increased in the serum of patients with osteoporosis. Together, miR-27a-3p contributed to diminished osteogenic function during osteogenic differentiation and might thus serve as a therapeutic target and diagnostic biomarker for osteoporosis.
\end{abstract}

\section{Introduction}

Osteoporosis is the most common disorder of bone remodeling in aging humans (1). It is associated with high health costs, and

Correspondence to: Professor Wei Zhang, Research Institute of Stomatology, Nanjing Medical University, Stomatological Hospital of Jiangsu Province, 136 Hanzhong Road, Nanjing, Jiangsu 210029, P.R. China

E-mail: sxm813121@163.com

*Contributed equally

Key words: microRNA-27a-3p, osteoporosis, osteogenic differentiation, osterix is characterized by an increased propensity to fragility fractures due to progressive loss of bone mass and bone quality, coupled with decreased osteoblast production and function $(2,3)$. In osteoporosis, bone homeostasis is disrupted by hormonal deficiency and aging, leading to increased bone turnover with enhanced bone formation and even greater rates of bone resorption, resulting in net bone loss $(4,5)$. Consequently, safe and effective strategies to stimulate osteoblast formation and activity are in great clinical demand. Anti-osteoporotic agents or food supplements that can stimulate new bone formation and improve trabecular microarchitecture should continue to be investigated for postmenopausal osteoporosis, as emerging therapies hold great promise (6-8).

MicroRNAs (miRNAs/miRs) are a diverse family of endogenous small non-coding RNAs (ncRNAs) that are $\sim 21$ nucleotides in length, which post-transcriptionally regulate the stability and translational efficiency of target mRNAs (9). miRNAs have been reported to contribute to each step of osteogenesis, starting from embryonic bone development to adult bone tissue maintenance, by regulating the growth, differentiation and functional activity of cells that constitute the bone tissue (10). In addition, substantial quantities of miRNAs are present in body fluids such as serum and plasma as circulating ncRNAs, and have been shown to play roles in cell communication by functioning as hormone-like molecules to influence the behaviors of different cells in a paracrine or endocrine manner $(11,12)$. Due to their high abundance and stability, circulating miRNAs have potential utility as non-invasive, blood-based biomarkers that can provide information on the disease and targeted therapies (13-21).

Icariin, a bioactive plant flavonoid, possesses the ability to stimulate the osteogenic differentiation of bone mesenchymal stem cells, improve osteoporosis in ovariectomized rats and induce bone repair in rabbits (22-27). In a previous study, it was demonstrated that icariin promoted osteogenic differentiation in MC3T3-E1 cells (28). The present study screened the differentially expressed miRNAs in icariin-treated MC3T3-E1 cells and found that miR-27a-3p expression was negatively associated with icariin treatment. Overexpression of miR-27a-3p inhibited osteoblast activity in vitro, as well as decreasing the expression levels of osterix (Osx). Furthermore, 
it was verified that Osx, a primary transcription factor required for osteoblast function, was a direct target of miR-27a-3p. Serum miR-27a-3p expression was validated in clinical osteoporosis specimens. Hence, the findings of the present study suggested that miRNAs could be used as a novel diagnostic tool for osteoporosis or serve as novel targets for therapeutic intervention.

\section{Materials and methods}

Cell culture. MC3T3-E1 preosteoblasts (obtained from the Cell Center of the Chinese Academy of Medical Sciences) were maintained in Minimum Essential Medium $\alpha$ ( $\alpha$-MEM; Gibco; Thermo Fisher Scientific, Inc.) supplemented with $10 \%$ fetal bovine serum (FBS; Gibco; Thermo Fisher Scientific, Inc.) and $1 \%$ penicillin/streptomycin (PS). 293T cells (American Type Culture Collection) were maintained in Dulbecco's modified Eagle's medium (DMEM; Gibco; Thermo Fisher Scientific, Inc.) supplemented with $10 \%$ FBS and $1 \%$ PS. Cultured cells were incubated at $37^{\circ} \mathrm{C}$ in a humidified chamber containing $5 \% \mathrm{CO}_{2}$.

Animals. Adult female C57BL/6 mice (weight, 17-20 g; age, 6-8 weeks; $n=3$ ) were purchased from the Model Animal Research Center of Nanjing University and maintained under standard animal housing conditions (12-h light/dark cycle and free access to food and water) in a temperature-controlled room $\left(24 \pm 1^{\circ} \mathrm{C}\right)$ with relative humidity $(50 \pm 10 \%)$ at the animal center of Nanjing Medical University. All procedures involving mice and the corresponding experimental protocols were approved by the Animal Care and Use Committee of Nanjing Medical University (approval no. IACUC-1706005). Following terminal anesthesia by intraperitoneal injection with pentobarbital sodium (100 mg/kg body weight), mice were euthanized by cervical dislocation and the death of mouse was verified 10 min by the following criteria: i) No breathing; ii) no nerve reflexes; iii) no heartbeat and iv) relaxed muscles. Then, the tissues (including the heart, liver, spleen, lung, kidney, bone and brain) were quickly dissected and separately immersed into liquid nitrogen.

RNA oligonucleotides and transfection assay. For transfection, $100 \mathrm{nM}$ inhibitors (5'-GCGGAACUUAGCCACUGU GAA-3'), mimics (5'-UUCACAGUGGCUAAGUUCCGC-3') and non-specific control oligonucleotides (5'-UUCUCCGAA CGUGUCACGUTT-3') of miR-27a-3p were obtained from Guangzhou RiboBio Co., Ltd. and transfected into cultured cells using Lipofectamine ${ }^{\circledR} 2000$ (Invitrogen, Thermo Fisher Scientific, Inc.), according to the manufacturer's instructions. At $48 \mathrm{~h}$ post-transfection, the cells were harvested and analyzed for mRNA and protein expression.

RNA isolation and reverse transcription-quantitative PCR $(R T-q P C R)$. Total RNA was isolated from cultured cells, as well as the tissues of C57BL/6 mice (including the heart, liver, spleen, lung, kidney, bone and brain) using RNAiso Plus according to the manufacturer's protocol (Takara Biotechnology Co., Ltd.). The ribosomal bands were visualized on a $1 \%$ TAE agarose gel to assess RNA integrity. cDNA was synthesized from $1 \mu \mathrm{g}$ total RNA in RT reactions using a
PrimeScript RT reagent kit (Takara Biotechnology Co., Ltd.), according to the manufacturer's instructions, and RT-qPCR was performed with SYBR Green reagents (Roche Diagnostics) on an ABI 7500 Real-Time PCR system (Applied Biosystems; Thermo Fisher Scientific, Inc.). The expression level of mature miR-27a-3p was determined by stem-loop RT-qPCR. U6 was used for normalization. The following primers sequences were used: miR-27a-3p, RT, 5'-GTCGTATCCAGTGCAGGG TCCGAGGTATTCGCACTGGATACGACGCGGAACT-3', forward, 5'-GCGGGCGTTCACAGTGGCTA-3' and reverse, 5'-CAGTGCAGGGTCCGAGGT-3'; U6, forward, 5'-CTC GCTTCGGCAGCACA-3' and reverse, 5'-AACGCTTCA CGAATTTGCGT-3'. The PCR amplification conditions were: $95^{\circ} \mathrm{C}$ for $5 \mathrm{~min}$, followed by 40 cycles of $95^{\circ} \mathrm{C}$ for $15 \mathrm{sec}$ and $60^{\circ} \mathrm{C}$ for $1 \mathrm{~min}$. The following primers sequences were used: Runt-related transcription factor 2 (Runx2), forward, 5'-ATG ATGACACTGCCACCTCTGAC-3' and reverse, 5'-AACTGC CTGGGGTCTGAAAAAGG-3'; Osx, forward, 5'-AGCGAC CACTTGAGCAAACAT-3' and reverse, 5'-GCGGCTGAT TGGCTTCTTCT-3'; alkaline phosphatase (ALP), forward, 5'-TGACCTTCTCTCCTCCATCC-3' and reverse, 5'-CTT CCTGGGAGTCTCATCCT-3'; osteocalcin (OC), forward, 5'-TGCTTGTGACGAGCTATCAG-3' and reverse, 5'-GAG GACAGGGAGGATCAAGT-3'; bone sialoprotein (BSP), forward, 5'-AAGCAGCACCGTTGAGTATGG-3' and reverse, 5'-CCTTGTAGTAGCTGTATTCATCCTC-3'; type I collagen (Col1 $\alpha 1$ ), forward, 5'-GCAACAGTCGCTTCACCTACA-3' and reverse, 5'-CAATGTCCAAGGGAGCCACAT-3'; and $\beta$-actin, forward, 5'-AGATGTGGATCAGCAAGCAG-3' and reverse, 5'-GCGCAAGTTAGGTTTTGTCA-3'. The relative expression levels were calculated using the $2^{-\Delta \Delta \mathrm{Cq}}$ method (29) and normalized to $\beta$-actin. For each data point, triplicate reactions were carried out and the experiment was repeated three times to assess statistical significance.

RT-PCR for Osx expression. Total RNA isolation and cDNA synthesis were conducted as described above. The amplification conditions for PCR were: $95^{\circ} \mathrm{C}$ for $5 \mathrm{~min}$, followed by 20-30 cycles of denaturation at $95^{\circ} \mathrm{C}$ for $15 \mathrm{sec}$, annealing at $56^{\circ} \mathrm{C}$ for $5 \mathrm{sec}$ and extension at $72^{\circ} \mathrm{C}$ for $30 \mathrm{sec}$. The PCR products were analyzed on $8 \%$ polyacrylamide gels and visualized using a Tanon 4100 gel imaging analysis system (Tanon). The primers for $\beta$-actin and Osx were same as those used for RT-qPCR.

Western blot analysis. Cells were lysed in RIPA buffer [50 mM Tris- $\mathrm{HCl}, \mathrm{pH}$ 7.5, $150 \mathrm{mM} \mathrm{NaCl}, 1 \%$ Nonidet P-40, $0.5 \%$ sodium deoxycholate and $0.1 \%$ sodium dodecyl sulfate (SDS)] plus protease inhibitors (Roche Diagnostics). Protein concentrations were determined using a Pierce ${ }^{\mathrm{TM}}$ BCA Protein Assay kit (Thermo Fisher Scientific, Inc.). Total protein $(20 \mu \mathrm{g})$ samples were boiled for $5 \mathrm{~min}$ in $1 \mathrm{X}$ loading buffer, incubated on ice, separated via 10\% SDS-PAGE and then transferred to PVDF membranes (EMD Millipore) with a semidry transfer apparatus (Bio-Rad Laboratories, Inc.). Non-specific protein interactions were blocked by incubation with $5 \%$ fat-free milk in TBS-Tween (TBST) buffer $(50 \mathrm{mM}$ Tris- $\mathrm{HCl}, 150 \mathrm{mM} \mathrm{NaCl}, 0.05 \%$ Tween 20, $\mathrm{pH}$ 7.6) for $1 \mathrm{~h}$ at room temperature. The membranes were incubated overnight with primary antibody in blocking buffer at $4^{\circ} \mathrm{C}$. Unbound 
antibody was removed by washing three times with TBST (10 $\mathrm{min} /$ wash). After rinsing, the membranes were incubated with horseradish peroxide (HRP)-conjugated goat anti-mouse (1:5,000; cat. no. sc-2005; Santa Cruz Biotechnology, Inc.) or HRP-conjugated goat anti-rabbit (1:5,000; cat. no. sc-2004; Santa Cruz Biotechnology, Inc.) secondary antibodies for $1 \mathrm{~h}$ at room temperature, followed by washing with TBST three times (10 $\mathrm{min} / \mathrm{wash})$. The immunoreactive bands were visualized with ECL reagent (Pierce; Thermo Fisher Scientific, Inc.). Densitometric analysis was performed using ImageJ software (version 1.49v; National Institutes of Health). Primary antibodies against Osx (1:4,000; cat. no. ab94744; Abcam) and $\beta$-actin (1:1,000; cat. no. sc-47778; Santa Cruz Biotechnology, Inc.) were used.

Plasmid construction and luciferase reporter assay. Osx cDNA was inserted into a pRK5 vector (Addgene, Inc.) at the EcoRI and HindIII sites to generate wild-type pRK5-Flag-Osx plasmid. Primer sequences for cloning the Osx CDS sequences were: Forward, 5'-CGGAATTCATGGCGTCCTCCCTGC TTGA-3' and reverse, 5'-CCCAAGCTTTCAGATCTCCAG CAAGTTGC-3'. Fragments of the Osx 3'-untranslated region (3'UTR) containing the $\mathrm{miR}-27 \mathrm{a}-3 \mathrm{p}$ recognition sequences were inserted behind the luciferase coding sequence at the $X b a \mathrm{I}$ site in the pGL3-promoter vector (Promega Corporation). Primer sequences for cloning the Osx 3'UTR sequences were: Wild-type (WT), forward, 5'-CTCTAGAGCTCCGACCTC CTCAACTT-3' and reverse, 5'-CTCTAGAAAGGCATT TCAAAGGCACA-3'; mutant (MUT), forward, 5'-GCCAGA AAGCTAGTAAACTTCAAGT-3' and reverse, 5'-ACTTGA AgTtTACTAGCTTTCTGGC-3'. The PrimeSTAR ${ }^{\circledR}$ Max DNA Polymerase (Takara Biotechnology Co., Ltd.) was used for the PCR, according to the manufacturer's protocol. The amplification conditions were as follows: $98^{\circ} \mathrm{C}$ for $3 \mathrm{~min}$; followed by 32 cycles of $98^{\circ} \mathrm{C}$ for $10 \mathrm{sec}$ and $60^{\circ} \mathrm{C}$ for $90 \mathrm{sec}$; and a final extension at $72^{\circ} \mathrm{C}$ for $10 \mathrm{~min}$. For the luciferase assay, $1 \times 10^{5} 293 \mathrm{~T}$ cells/well were co-transfected in 24 -well plates with the above reporter constructs $(0.2 \mu \mathrm{g})$ along with phRL-null $(0.01 \mu \mathrm{g}$; Renilla plasmid for normalization; Promega Corporation) and $100 \mathrm{nM}$ control mimic (miR-C) or miR-27a-3p mimic using Lipofectamine ${ }^{\circledR} 2000$. The cells were collected $48 \mathrm{~h}$ after transfection and the luciferase activities were measured with a Dual-luciferase Reporter Assay system (Promega Corporation) and a GloMax ${ }^{\mathrm{TM}}$ Base instrument (Promega Corporation). The firefly luciferase activity was normalized to Renilla luciferase activity.

Bioinformatics prediction and conservative analysis. Potential targets of miR-27a-3p were predicted from three different algorithms: TargetScan Mouse v.6.2 (http://www.targetscan.org), miRanda (http://www.microrna. org/microrna/home.do) and RNA22 (https://cm.jefferson.edu/ rna22v1.0-mus_musculus/GetInputs.jsp). TargetScan predicts the biological targets of miRNAs by searching for the presence of conserved $8 \mathrm{mer}, 7 \mathrm{mer}$ and 6 mer sites that match the seed region of each miRNA. Potential target mRNAs were selected if they were predicted by $\geq 2$ algorithms. The mature sequences for mmu-miR-27a-3p and hsa-miR-27a-3p were obtained from miRBase (http://www.mirbase.org/) and aligned by ClustalX (http://www.clustal.org).
Table I. Age distribution of the patients and controls.

\begin{tabular}{lcc}
\hline Age, years & Normal & Osteoporosis \\
\hline $50-59$ & 9 & 5 \\
$60-69$ & 31 & 32 \\
$70-79$ & 8 & 32 \\
$80-90$ & 4 & 16 \\
\hline
\end{tabular}

ALP staining. To induce osteogenic differentiation, cells were cultured in differentiation-inducing medium containing $50 \mathrm{mg} / \mathrm{l}$ ascorbic acid, $10 \mathrm{mM} \beta$-glycerophosphate and $10 \mathrm{nM}$ dexamethasone for the indicated times with regular medium changes. For ALP staining, after induction for 5-7 days, cells were fixed with $95 \%$ ethanol and stained with BCIP/NBT solution according to the manufacturer's protocol (Beyotime Institute of Biotechnology) at room temperature for $2 \mathrm{~h}$. The ALP-positive cells were stained blue/purple. Stained cells were visualized using the Canon IXUS210 camera (Canon, Inc.; magnification, x5). ImageJ version 1.49 software (National Institutes of Health) was used for image analysis and quantification.

Icariin treatment. Icariin (94.2\% purity) was purchased from the National Institutes for Food and Drug Control. Stock solutions of Icariin $(10 \mathrm{mM})$ were prepared in DMSO $(\geq 99.7 \%$; Sigma-Aldrich; Merck $\mathrm{KGaA}$ ) and stored at $-20^{\circ} \mathrm{C}$. Icariin treatment was performed as previously described (28). Briefly, MC3T3-E1 cells were cultured in differentiation-inducing medium containing $50 \mathrm{mg} / 1$ ascorbic acid, $10 \mathrm{mM}$ $\beta$-glycerophosphate and $10 \mathrm{nM}$ dexamethasone, and $5 \mu \mathrm{M}$ Icariin for $48 \mathrm{~h}$ at $37^{\circ} \mathrm{C}$ in a humidified chamber containing $5 \% \mathrm{CO}_{2}$.

Clinical samples. This study conformed to the principles of the Declaration of Helsinki and was approved by the Ethics Committee of Jiangsu Province Hospital of Chinese Medicine. A total of 137 female participants were recruited for this study, including 85 participants with osteoporosis (50-90 years old) and 52 healthy participants (50-90 years old) from the Jiangsu Province Hospital of Chinese Medicine and Northern Jiangsu People's Hospital between February 2014 and April 2019 (Table I). Each subject underwent a clinical examination, and routine biochemical tests were performed to exclude subjects with systemic and metabolic bone disorders other than osteoporosis. Assessment of the most susceptible sites of osteoporotic fractures, such as the lumbar spine and the head of the femur, by dual X-ray absorptiometry was used as the reference method for measuring total bone mineral density (BMD). The osteoporosis group was defined as BMD T-scores $\leq-2.5$ at the lumbar. Informed consent was obtained from all participants prior to sample collection. Clinical samples were obtained from the participants, including local health volunteers and patients who were referred to the hospital, who either presented at the bone clinic or the Department of Radiology for a BMD scan. Serum samples were prepared by centrifugation at 2,500 x g for $30 \mathrm{~min}$ at room temperature. Aliquots of the supernatants were frozen at $-80^{\circ} \mathrm{C}$ until RNA extraction. 
Statistical analyses. Experimental data were analyzed by Student's t-test or one-way analysis of variance with Bonferroni's correction using GraphPad Prism 6.0 (GraphPad Software, Inc.). All pairs of columns were compared, and the bars denote the mean \pm standard deviation from 3 independent experiments. $\mathrm{P}<0.05$ was considered to indicate a statistically significant difference.

\section{Results}

miR-27a-3p expression is downregulated during osteogenic differentiation. Our previous study demonstrated that icariin treatment significantly elevated the gene expression of osteogenic markers and increased ALP activity, thus promoting osteogenic differentiation of MC3T3-E1 cells (28). The present study first examined the levels of miR-27a-3p during icariininduced osteogenic differentiation. Results demonstrated that the expression of osteogenic marker genes such as Runx 2 and Osx was rapidly increased in MC3T3-E1 cells treated with icariin $(5 \mu \mathrm{M})$ for $48 \mathrm{~h}$ (Fig. 1A), but that of miR-27a-3p was significantly downregulated (Fig. 1B). Notably, miR-27a-3p, encoded by a gene located on mouse chromosome 1, was located at a noncoding region and the precursor sequences displayed the characteristic miRNA precursor stem-loop secondary structure according to the miRNA repository miRBase (Fig. 1C). To further evaluate the function of miR-27a-3p, its tissue distribution in normal adult mice was examined by RT-qPCR. The expression of mature miR-27a-3p was detected in all seven types of tissues, including the heart, liver, spleen, lung, kidney, bone and brain. In addition, results demonstrated that miR-27a-3p was preferentially expressed in the lung and bone tissues (Fig. 1D). Analysis by ClustalX demonstrated that miR-27a-3p was highly conserved in both Homo sapiens and Mus musculus (Fig. 1E).

To determine the role of miR-27a-3p in osteoblast lineage commitment, its expression level was detected by culturing MC3T3-E1 preosteoblasts in osteogenic medium for different time periods. As shown in Fig. 1F, miR-27a-3p expression in MC3T3-E1 cells began to decrease over the course of osteogenic differentiation (from days 3 to 12). The expression levels of Runx2 and Osx increased gradually starting from day 3 , reaching a maximum on day 7 ; however, their levels started to decline during the terminal differentiation phase. Together, these findings suggest that miR-27a-3p may function as a miRNA that modulates osteogenic differentiation.

Osx is a direct target of miR-27a-3p. To establish the link between miR-27a-3p and osteoblast function, putative protein targets involved in osteogenic differentiation were screened. Osx, an osteoblast-specific transcription factor, was selected as a target for validation. Single putative miR-27a-3p recognition sites were predicted within the Osx 3'UTR sequences by TargetScan Mouse v.6.2 and RNA22. According to the sequence analysis, Osx contained a 7-nucleotide site within its 3'UTR that matched the seed region of miR-27a-3p (Fig. 2A). Furthermore, tissue distribution analysis demonstrated that the Osx level was highest in the bone (Fig. 2B).

To gain insight into the mechanism via which miR-27a-3p modulates Osx expression, the 3'UTR of the Osx gene containing one miR-27a-3p binding site was fused downstream of a luciferase reporter (Fig. 2C). Results demonstrated that miR-27a-3p mimic significantly inhibited the luciferase reporter activity of the WT Osx 3'UTR compared with miR-C (Fig. 2D). Another luciferase reporter was constructed with an Osx 3'UTR containing mutant sequences of the miR-27a-3p binding site (Fig. 2C). The luciferase reporter activity of the MUT Osx 3'UTR was not suppressed by miR-27a-3p mimic (Fig. 2D). Taken together, introduction of mutations in these sequences abolished the ability of miR-27a-3p to inhibit reporter activity, confirming the selective interaction of miR-27a-3p with mRNAs and indicating that the single recognition element identified in the $3^{\prime}$ UTR of the Osx mRNA is sufficient for miR-27a-3p activity. Accordingly, transfection with miR-27a-3p mimic alone decreased the amount of endogenous Osx protein in cultured cells (Fig. 2E), but had no significant effect on Osx mRNA levels (Fig. 2F), suggesting that miR-27a-3p downregulates Osx expression by inhibiting its translation.

$m i R-27 a-3 p$ regulates osteoblast activity in vitro. Due to the role of miR-27a-3p in regulating Osx expression, the physiological effect of miR-27a-3p overexpression on osteogenic differentiation was evaluated in vitro. Results demonstrated that miR-27a-3p levels were substantially upregulated after transfection with miR-27a-3p mimic (Fig. 3A). In addition, the expression of osteoblast marker genes, including ALP, OC, BSP and Coll $\alpha 1$, which are Osx downstream genes, was downregulated in MC3T3-E1 cells transfected with miR-27a-3p mimic compared to that in cells transfected with control mimic (Fig. 3B). Conversely, the expression of these osteoblast marker genes was upregulated in MC3T3-E1 cells transfected with miR-27a-3p inhibitor (Fig. 3C and D). Consistent with the changes in osteoblast marker gene expression, weakened ALP staining was observed in MC3T3-E1 cells transfected with miR-27a-3p mimic compared to that in cells transfected with control mimic (Fig. 3E).

Osx rescues the effect of $m i R-27 a-3 p$ on osteogenic differentiation. Having demonstrated that miR-27a-3p suppresses osteogenic differentiation, it was next assessed whether overexpression of Osx could rescue the effect of miR-27a-3p on osteogenic differentiation in vitro. As shown in Fig. 4A, Osx protein level was enhanced after transfection with Flag-Osx expression plasmid in 293T cells. Of note, when co-transfected with miR-27a-3p mimic and Osx overexpression plasmid, the miR-27a-3p-induced inhibition of osteogenic differentiation was attenuated (Fig. 4B and C). These results suggested that the predominant effect of miR-27a-3p on osteogenic differentiation was mediated via the downregulation of Osx.

Circulating miR-27a-3p level is associated with osteoporosis. Based on BMD, 137 participants were classified into 2 groups: Group A, 52 (female) non-osteoporotic controls; and group B, 85 (female) patients with osteoporosis. Circulating miRNAs from serum samples were tested using RT-qPCR. The results demonstrated that miR-27a-3p levels were significantly elevated in patients with osteoporosis compared with in non-osteoporotic controls (Fig. 5). These data suggested that elevated levels of circulating miR-27a-3p in clinical samples may be associated with the development of osteoporosis in patients. 
A
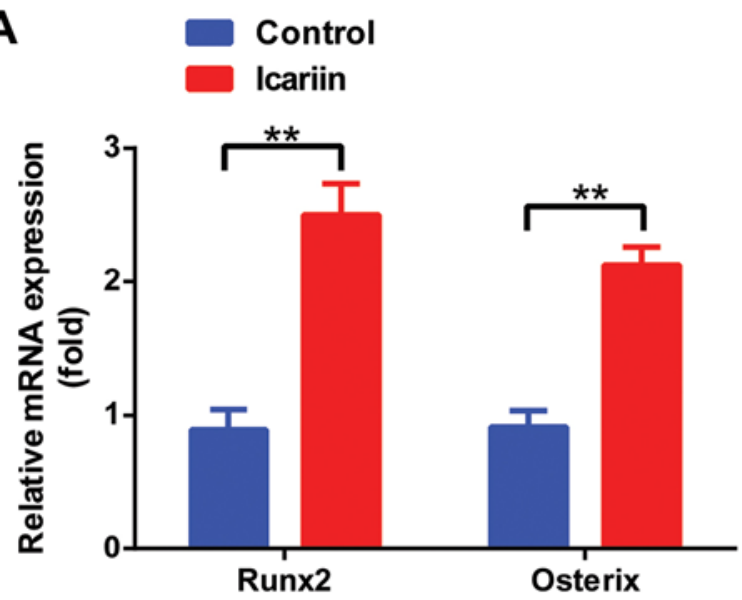

C

miR-27a-3p

u - ua a a ug u a uccac

gg cc ggg gc gggcuuagc cu gugagca gg a

cc gg c cc cg cuugaaucg ga cacuugu cu $\mathrm{g}$

c a $u \cdot c$ c gu $\cdot \quad$ g gaaac

B

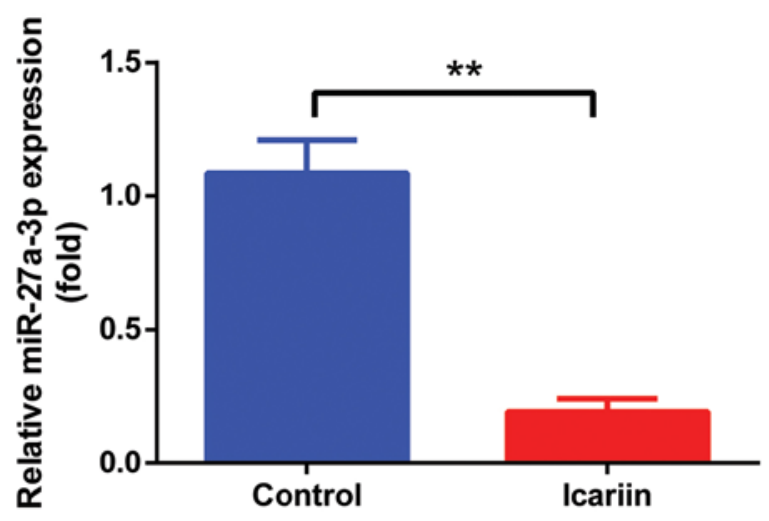

D

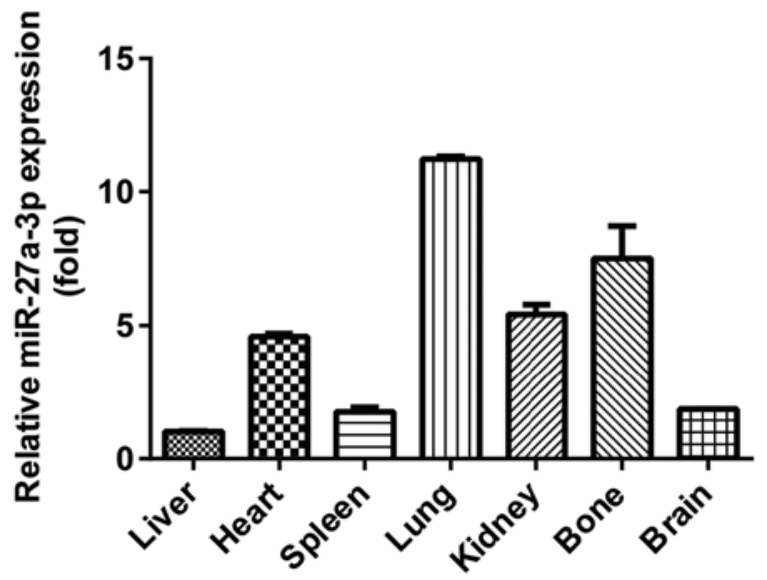

$\mathbf{F}$

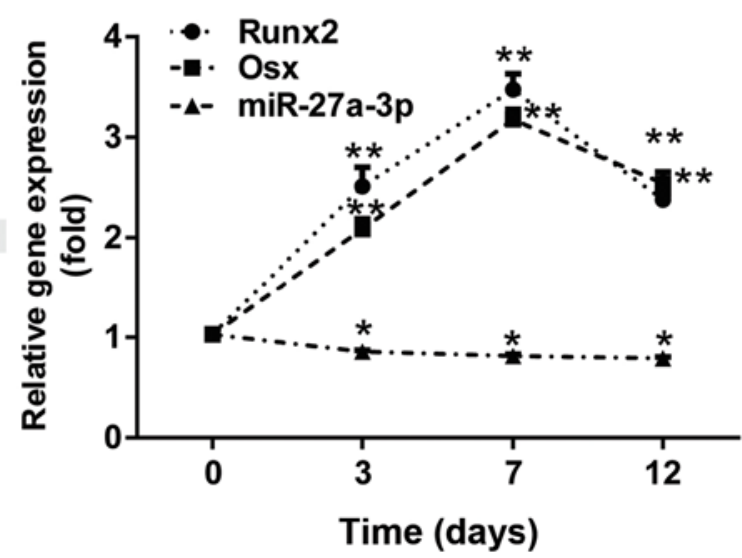

Figure 1. miR-27a-3p expression is downregulated during osteogenic differentiation. (A) Relative mRNA levels of Runx2 and Osx assayed by RT-qPCR in MC3T3-E1 cells treated with icariin $(5 \mu \mathrm{M})$ for $48 \mathrm{~h}$. (B) RT-qPCR detection of miR-27a-3p levels in MC3T3-E1 cells treated with icariin $(5 \mu \mathrm{M})$ for $48 \mathrm{~h}$. ${ }^{* *} \mathrm{P}<0.01$. (C) Schematic diagram of the secondary structure of pre-miR-27a. The 22-nucleotide mature miR-27a-3p is indicated in red. (D) RT-qPCR detection of miR-27a-3p level in each tissue from mice. Total RNA was isolated from the tissues. (E) Sequence alignment of miR-27a-3p between mice and humans. (F) RT-qPCR analysis of the time course changes of Osx and Runx2 mRNA levels and miR-27a-3p level in MC3T3-E1 cells cultured in osteogenic differentiation medium for 12 days. U6 was used as the internal control. ${ }^{*} \mathrm{P}<0.05,{ }^{* *} \mathrm{P}<0.01$ vs. 0 days. miR, microRNA; Runx 2 , runt-related transcription factor 2 ; Osx, osterix; RT-qPCR, reverse transcription-quantitative PCR.

\section{Discussion}

Osteoporosis is one of the major diseases caused by age- or disease-related bone loss (30). A healthy mammalian skeleton is maintained by constant bone remodeling, an active coupling process involving bone-forming osteoblasts and bone-resorbing osteoclasts $(5,30)$. MC3T3-E1 cells, preosteoblasts that mature into preosteocytes in a mineralized matrix, have been stimulated with icariin to induce osteogenic differentiation (28). The present study demonstrated that miR-27a-3p expression was rapidly downregulated during icariin-induced osteogenic differentiation in MC3T3-E1 cells. 
A \begin{tabular}{l|l} 
Mouse & CCCAGCCAGAAAGCUGUGAAACUUCAAGUCC \\
Human & CCCAGCCAG -AAGCUGUGAAACCUCAAGUCC \\
Rat & CCCCAGCCAG -AAGCUGUGAAACUUCAAGUCC \\
Guinea pig & CCCCAGCCAG -AAGCUGUGAAACCUCAAGUCC \\
Chimpanzee & CCCCAGCCAG -AAGCUGUGAAACCUCAAGUCC \\
Rhesus & CCCCAGCCAG -AAGCUGUGAAACCUCAAGUCC \\
Shrew & CCUUGGCCAG -AAGCUGUGAAUCUGAGUAC \\
Hedgehog & CCCUGGCCAG -AAGCUGUGAAGCCUCAAUCC \\
Dog & CCCCAGCCAG -AAGCUGUGAAACCUCGAGUCC \\
Cat & CCCCAGCCAG -AAGCUGUGAAACCUCAAGCCC \\
Horse & CCCCAGCCAG -AAGCUGUGAAACCUCAAGUCC \\
Cow & CCCUAGCCAG -AAGCUGUGAAAACUCAAGCCC \\
Armadillo & CCCCAGCCAG -AAGCUGUGAAACCACAAGUCC \\
Tenrec & CCCCAGCCAG -AAGCUGUGAAACUUCAAGUCC
\end{tabular}

C

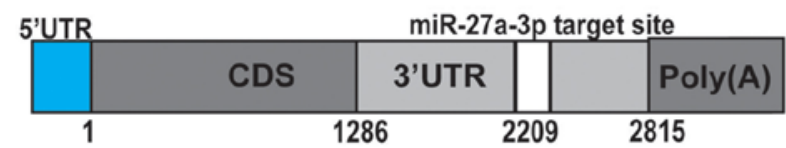

\section{Osx-3'UTR-WT}

mmu-miR-27a-3p

Osx-3'UTR-MUT
5' ...GCCCCAGCCAGAAAGCUGUGAAA...3' | || ||| 3' CgccuUgaAucggugacacuU 5' II I 5 '...GCCCCAGCCAGAAAGCUAGTAAA....3'
B
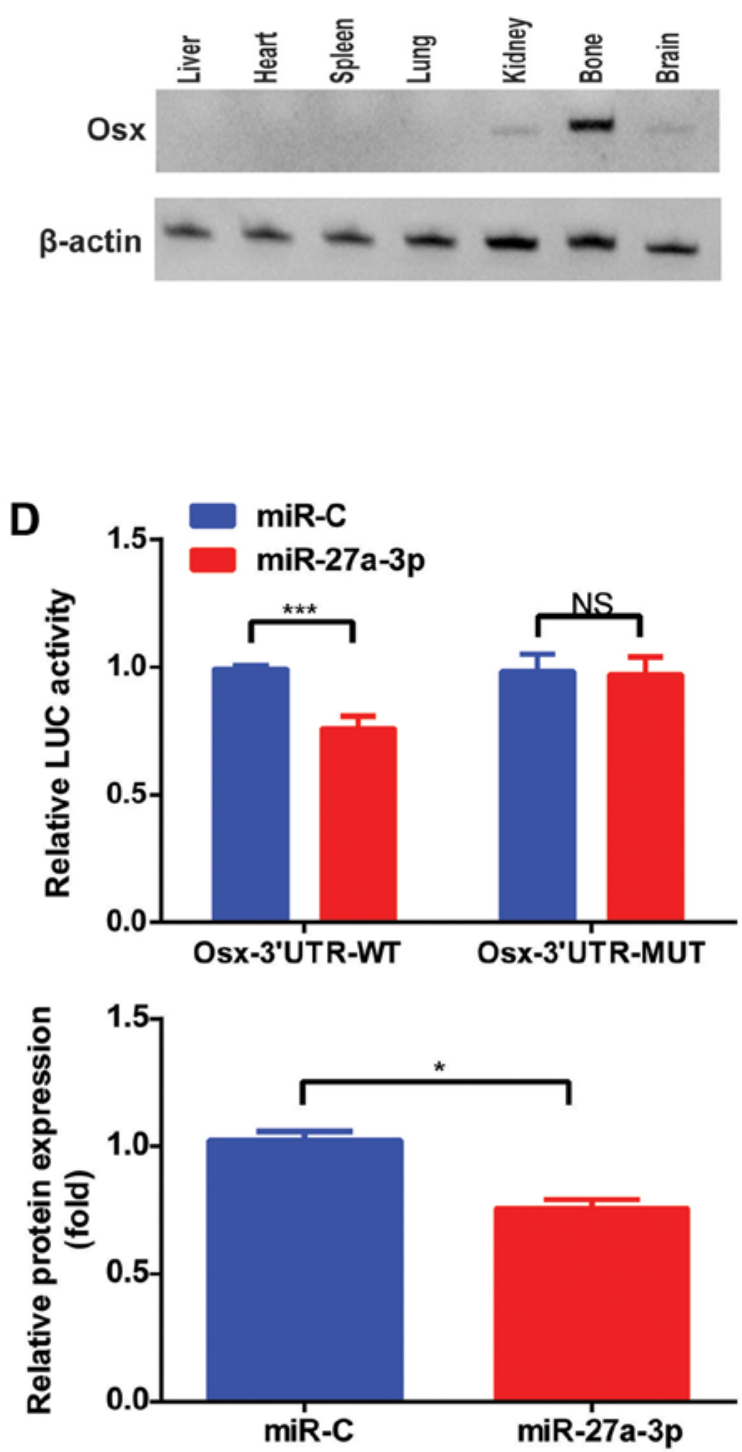

$\mathbf{F}$ miR-C miR-27a-3p
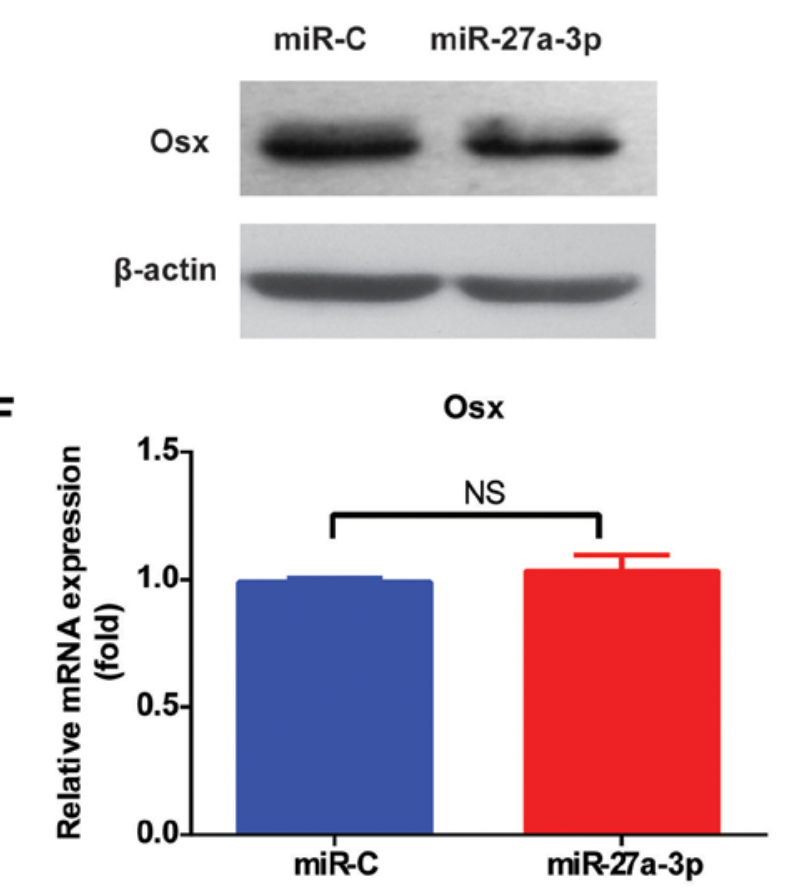

E

Figure 2. Osx is a direct target of miR-27a-3p. (A) Sequence alignment of the Osx 3'UTR by TargetScan. (B) RT-qPCR detection of Osx expression in each tissue from mice. (C) Schematic illustration of the design of the luciferase reporter construct with WT Osx 3'UTR or site-directed mutant Osx 3'UTR. (D) Luciferase activity of $293 \mathrm{~T}$ cells co-transfected with WT or mutant Osx 3'UTR reporter and miR-27a-3p mimic or miR-C. The relative luciferase activity, defined as the ratio of 3'UTR reporter activity to that of the internal control (Renilla), was determined $48 \mathrm{~h}$ after transfection. (E) Western blot analysis and quantification of Osx protein levels after transfection with miR-27a-3p mimic or miR-C in MC3T3-E1 cells. (F) Effects of miR-27a-3p mimic or control on Osx mRNA levels in MC3T3-E1 cells, as determined by RT-qPCR. "P<0.05, ${ }^{* * *} \mathrm{P}<0.001$. Osx, osterix; miR, microRNA; miR-C, mimic control; RT-qPCR, reverse transcription-quantitative PCR; WT, wild-type; MUT, mutant; 3'UTR, 3'-untranslated region; NS, not significant.

A growing body of evidence has revealed that miR-27a-3p acts as a key regulator of bone biology (31-36). Compared to 293T cells, MG63 cells and osteogenic hFOBs cells have been shown to display higher levels of miR-27a due to osteogenesis (34). 
A

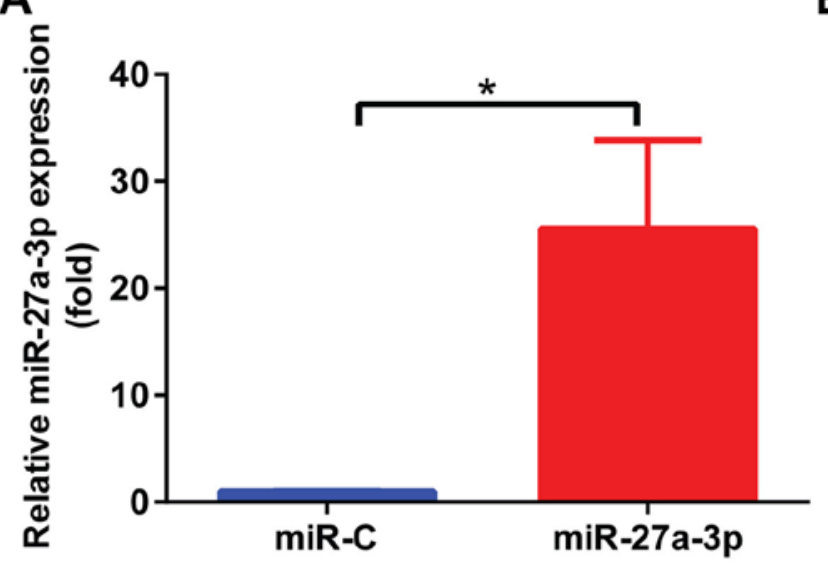

C

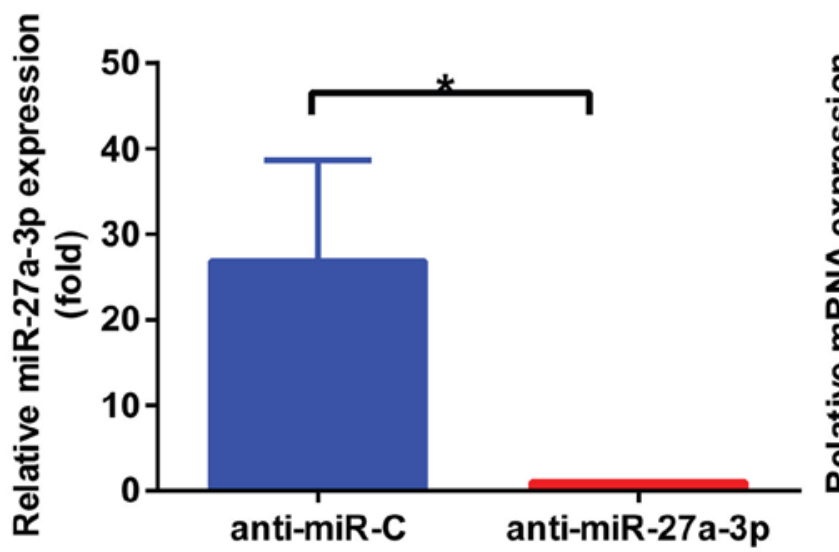

E

Mock

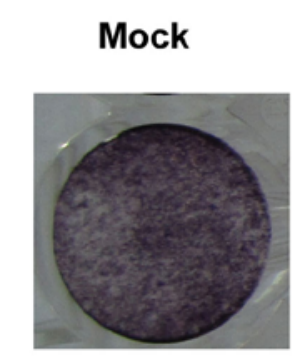

B

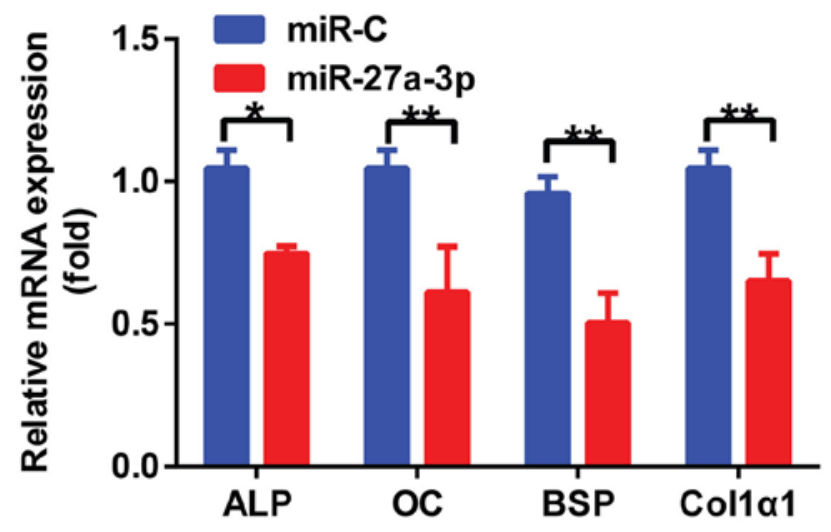

D

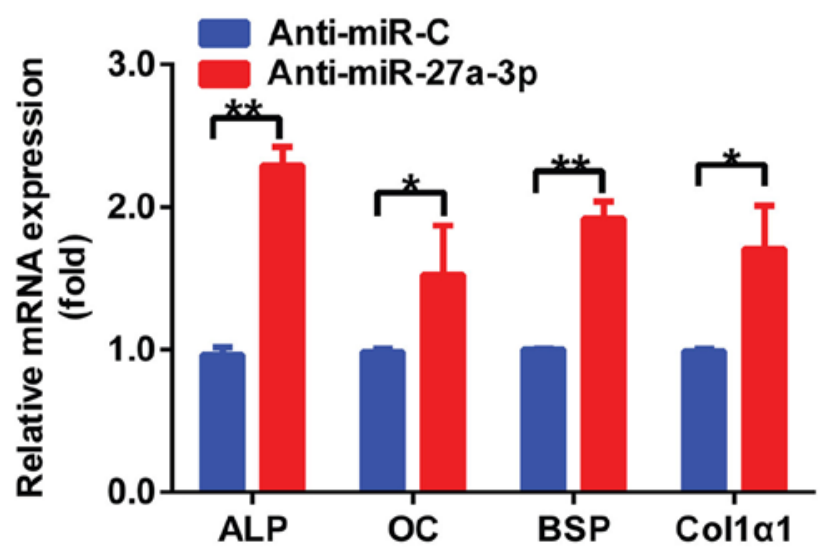

miR-C
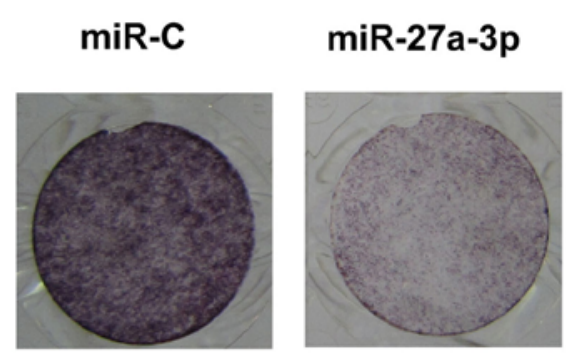

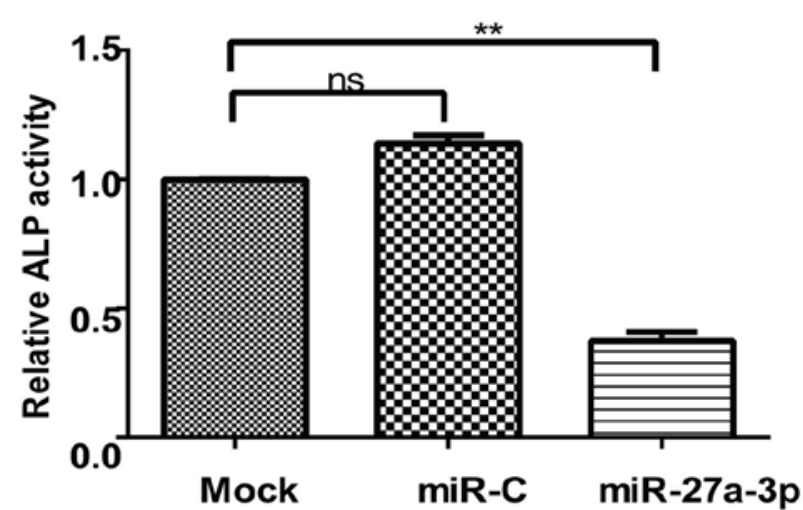

Figure 3. miR-27a-3p regulates osteoblast activity in vitro. (A) Expression of miR-27a-3p in MC3T3-E1 cells transfected with miR-27a-3p mimic or miR-C for $48 \mathrm{~h}$, as determined by RT-qPCR. U6 was used as the internal control. (B) RT-qPCR detection of the levels of osteoblast marker genes in MC3T3-E1 cells transfected with miR-27a-3p mimic or miR-C for 48 h. $\beta$-actin was used as the internal control. (C) Expression of miR-27a-3p in MC3T3-E1 cells transfected with miR-27a-3p inhibitor or anti-miR-C for $48 \mathrm{~h}$, as determined by RT-qPCR. U6 was used as the internal control. (D) RT-qPCR detection of the levels of osteoblast marker genes in MC3T3-E1 cells transfected with miR-27a-3p inhibitor or control for $48 \mathrm{~h}$. $\beta$-actin was used as the internal control. (E) ALP staining results after induction of osteogenic differentiation for 7 days and quantification of ALP activity. ${ }^{*} \mathrm{P}<0.05$, ${ }^{* *} \mathrm{P}<0.01$. miR, microRNA; miR-C, control mimic; anti-miR-C, control inhibitor; RT-qPCR, reverse transcription-quantitative PCR; ALP, alkaline phosphatase; OC, osteocalcin; BSP, bone sialoprotein; Coll $\alpha 1$, type I collagen.

In addition, the inhibitory effects of agomiR-27a-3p on the expression of Runx2, Osx, OC and Colla1 in periodontal ligament stem cells have been demonstrated (35). Overexpression of miR-27a has been shown to weaken ALP and alizarin red staining during osteogenic differentiation of Satb2-induced bone marrow stromal cells (36). Consistent with the inhibitory role of miR-27a-3p in osteogenic differentiation described by these studies, the present study found that the expression of osteogenic marker genes and ALP activity were significantly decreased in miR-27a-3p-overexpressing MC3T3-E1 cells 
A

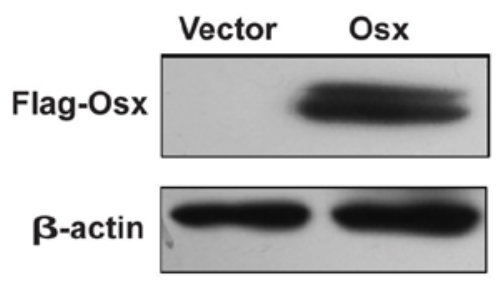

C

miR-C+Vector miR-27a-3p+Vector miR-27a-3p+Osx
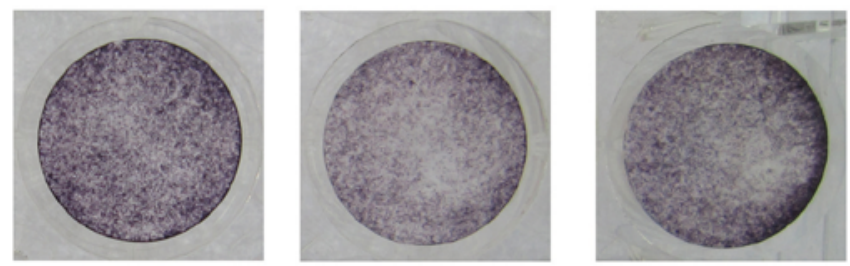

B
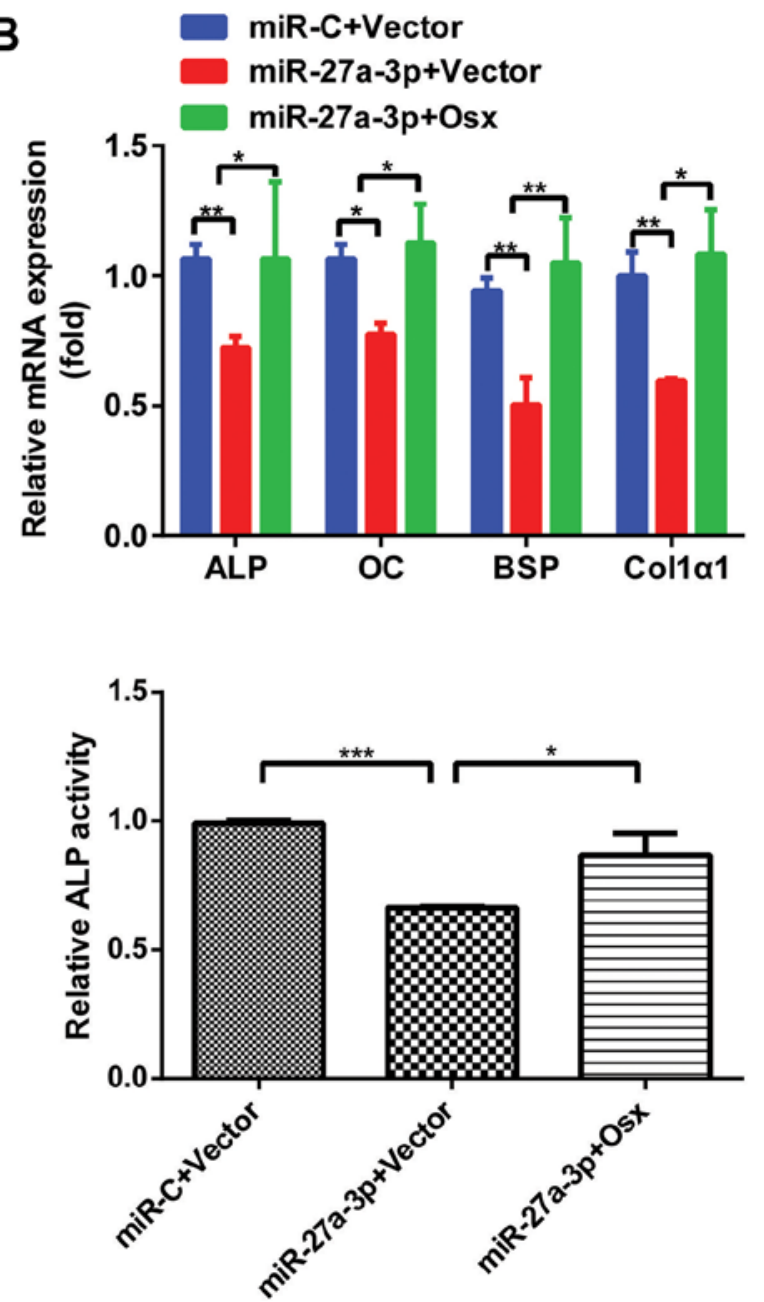

Figure 4. Osx rescues the effect of miR-27a-3p on osteogenic differentiation. (A) Western blot analysis of Osx protein level after transfection with Flag-Osx expression plasmid or vector alone in $293 \mathrm{~T}$ cells. $\beta$-actin was used as the internal control. (B) Expression of osteoblast marker genes, as determined by reverse transcription-quantitative PCR following miR-27a-3p-mediated inhibition of osteogenic differentiation in MC3T3-E1 cells with restored expression of Osx. $\beta$-actin was used as the internal control. (C) ALP staining analysis after miR-27a-3p-mediated inhibition of osteogenic differentiation in MC3T3-E1 cells with restored Osx expression and quantification of ALP activity. ${ }^{*} \mathrm{P}<0.05,{ }^{* *} \mathrm{P}<0.01,{ }^{* * *} \mathrm{P}<0.001$. miR, microRNA; miR-C, control mimic; Osx, osterix; ALP, alkaline phosphatase; OC, osteocalcin; BSP, bone sialoprotein; Coll $\alpha 1$, type I collagen.

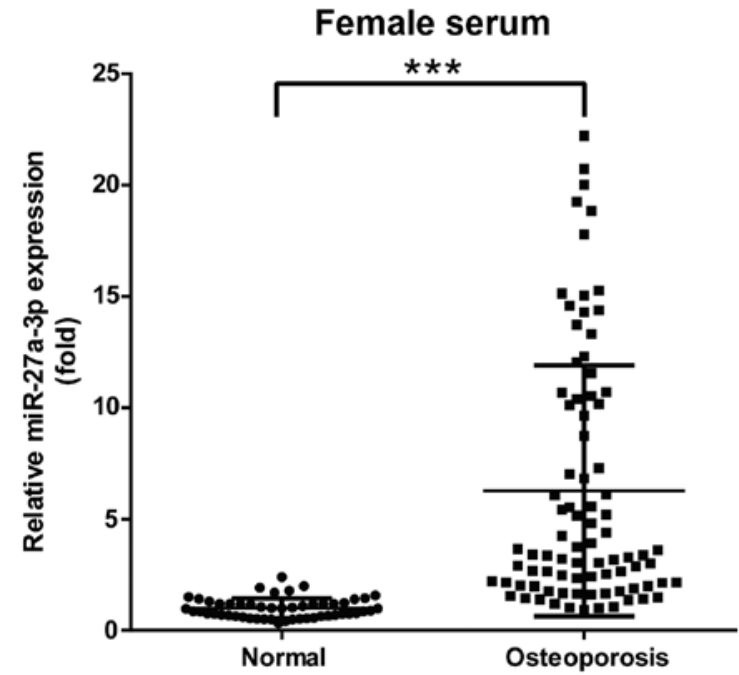

Figure 5. Circulating miR-27a-3p level is associated with osteoporosis. Circulating miR-27a-3p expression levels in the serum of female patients with osteoporosis $(\mathrm{n}=85)$ compared with female non-osteoporotic controls $(\mathrm{n}=52)$, as determined by reverse transcription-quantitative PCR. U6 was used as the internal control. ${ }^{* * *} \mathrm{P}<0.001$. miR, microRNA. in vitro. These effects could be reversed by suppression of miR-27a-3p, suggesting that miR-27a-3p is a negative regulator of osteogenic differentiation in MC3T3-E1 cells.

To explore the molecular mechanisms via which miR-27a-3p regulates the osteogenic differentiation of MC3T3-E1 cells, the potential targets of miR-27a-3p were predicted using miRNA target prediction tools. Osx was identified as a potential target of miR-27a-3p. The present study demonstrated that miR-27a-3p directly targeted Osx by binding to its $3^{\prime}$ UTR. Osx, a zinc-finger-containing transcription factor, acts downstream of Runx2, and regulates the differentiation and/or function of osteoblasts (37). Our previous study revealed that Osx expression in $\mathrm{C} 2 \mathrm{C} 12$ cells is regulated by miR-214, a suppressor of osteogenic differentiation (38). The present study reported that Osx is a direct target of miR-27a-3p and is involved in osteogenic differentiation. Of note, when Osx was overexpressed, osteogenic marker gene expression and ALP activity were restored in miR-27a-3p-overexpressing MC3T3-E1 cells. This indicated that miR-27a-3p inhibited osteogenic differentiation via Osx. Based on these data, it was hypothesized that miR-27a-3p was capable of inhibiting osteogenic differentiation. 
In postmenopausal osteoporosis, estrogen therapy effectively prevents bone loss (39). Emerging evidence has demonstrated that estrogen exhibits an inhibitory role in osteoclast formation and enhances osteoblast function $(40,41)$. During estrogen-decreased osteoclast differentiation, miR-27a remarkably enhances the inhibitory effect of estrogen (40). In addition, following treatment with antiosteoporotic agents, serum miR-27a levels are significantly reduced in postmenopausal women (42). The present study measured the serum levels of miR-27a-3p in 85 samples with osteoporosis and found that the miR-27a-3p levels were significantly higher in osteoporotic patients compared with those in non-osteoporotic controls. These findings implied that miR-27a-3p may serve as a promising marker for the detection of osteoporosis.

Taken together, the results of the present study demonstrated that miR-27a-3p suppressed osteogenic differentiation via downregulating Osx expression. The findings revealed the regulatory role of miR-27a-3p in osteogenic differentiation, and suggested that therapies targeting miR-27a-3p may serve as a promising strategy for the treatment of osteoporosis. The results of the present study provided novel insight into the mechanism by which miRNAs regulate bone physiology.

\section{Acknowledgements}

Not applicable.

\section{Funding}

This work was supported by grants from the Natural Science Foundation of Jiangsu Province (grant nos. BK20171057, BK20171489 and BK20161323), the National Natural Science Foundation of China (grant nos. 81500823, 81570804 and 81873105).

\section{Availability of data and materials}

The datasets used and/or analyzed during the current study are available from the corresponding author on reasonable request.

\section{Authors' contributions}

YX, DL and ZZ designed the experiments. LL onducted experiments on the clinical samples and provided material support.. YX, DL and ZZ performed most of the experiments. YX drafted the manuscript. CM performed the bioinformatics analysis. YJ contributed to the analysis and interpretation of the data. WZ performed the animal experiments and modified the manuscript. All authors read and approved the final manuscript.

\section{Ethics approval and consent to participate}

Informed consent was obtained from all participants. Human studies conformed to the principles of the Declaration of Helsinki and were approved by the Ethics Committee of Jiangsu Province Hospital of Chinese Medicine. All procedures involving mice and the corresponding experimental protocols were approved by the Animal Care and Use Committee of Nanjing Medical University (approval no. IACUC-1706005).

\section{Patient consent for publication}

Not applicable.

\section{Competing interests}

The authors declare that they have no competing interests.

\section{References}

1. Richards JB, Zheng HF and Spector TD: Genetics of osteoporosis from genome-wide association studies: Advances and challenges. Nat Rev Genet 13: 576-588, 2012.

2. Black DM and Rosen CJ: Clinical practice. Postmenopausal osteoporosis. N Engl J Med 374: 254-262, 2016.

3. Kim KM, Park SJ, Jung SH, Kim EJ, Jogeswar G, Ajita J, Rhee Y, Kim CH and Lim SK: MiR-182 is a negative regulator of osteoblast proliferation, differentiation, and skeletogenesis through targeting FoxO1. J Bone Miner Res 27: 1669-1679, 2012.

4. Yu B, Chang J, Liu Y, Li J, Kevork K, Al-Hezaimi K, Graves DT, Park NH and Wang CY: Wnt4 signaling prevents skeletal aging and inflammation by inhibiting nuclear factor- $\kappa \mathrm{B}$. Nat Med 20: 1009-1017, 2014.

5. Long F: Building strong bones: Molecular regulation of the osteoblast lineage. Nat Rev Mol Cell Biol 13: 27-38, 2011.

6. Mulder JE, Kolatkar NS and LeBoff MS: Drug insight: Existing and emerging therapies for osteoporosis. Nat Clin Pract Endocrinol Metab 2: 670-680, 2006.

7. Guo AJ, Choi RC, Cheung AW, Chen VP, Xu SL, Dong TT, Chen JJ and Tsim KW: Baicalin, a flavone, induces the differentiation of cultured osteoblasts: An action via the Wnt/beta-catenin signaling pathway. J Biol Chem 286: 27882-27893, 2011.

8. Reid IR: Short-term and long-term effects of osteoporosis therapies. Nat Rev Endocrinol 11: 418-428, 2015.

9. Chang TC, Yu D, Lee YS, Wentzel EA, Arking DE, West KM, Dang CV, Thomas-Tikhonenko A and Mendell JT: Widespread microRNA repression by Myc contributes to tumorigenesis. Nat Genet 40: 43-50, 2008

10. Lian JB, Stein GS, van Wijnen AJ, Stein JL, Hassan MQ, Gaur T and Zhang Y: MicroRNA control of bone formation and homeostasis. Nat Rev Endocrinol 8: 212-227, 2012.

11. Anfossi S, Babayan A, Pantel K and Calin GA: Clinical utility of circulating non-coding RNAs-an update. Nat Rev Clin Oncol 15: 541-563, 2018

12. Hackl M, Heilmeier U, Weilner S and Grillari J: Circulating microRNAs as novel biomarkers for bone diseases-complex signatures for multifactorial diseases? Mol Cell Endocrinol 432: 83-95, 2016.

13. Zeng HC, Bae Y, Dawson BC, Chen Y, Bertin T, Munivez E, Campeau PM, Tao J, Chen R and Lee BH: MicroRNA miR-23a cluster promotes osteocyte differentiation by regulating TGF- $\beta$ signalling in osteoblasts. Nat Commun 8: 15000, 2017.

14. Li D, Liu J, Guo B, Liang C, Dang L, Lu C, He X, Cheung HY, $\mathrm{Xu} \mathrm{L}, \mathrm{Lu} \mathrm{C}$, et al: Osteoclast-derived exosomal miR-214-3p inhibits osteoblastic bone formation. Nat Commun 7: 10872, 2016.

15. Seeliger C, Karpinski K, Haug AT, Vester H, Schmitt A, Bauer JS and van Griensven M: Five freely circulating miRNAs and bone tissue miRNAs are associated with osteoporotic fractures. J Bone Miner Res 29: 1718-1728, 2014.

16. Weilner S, Schraml E, Wieser M, Messner P, Schneider K, Wassermann K, Micutkova L, Fortschegger K, Maier AB, Westendorp R, et al: Secreted microvesicular miR-31 inhibits osteogenic differentiation of mesenchymal stem cells. Aging Cell 15: 744-754, 2016.

17. Weilner S, Skalicky S, Salzer B, Keider V, Wagner M, Hildner F, Gabriel C, Dovjak P, Pietschmann P, Grillari-Voglauer R, et al: Differentially circulating miRNAs after recent osteoporotic fractures can influence osteogenic differentiation. Bone 79: 43-51, 2015.

18. Mandourah AY, Ranganath L, Barraclough R, Vinjamuri S, Hof RV, Hamill S, Czanner G, Dera AA, Wang D and Barraclough DL: Circulating microRNAs as potential diagnostic biomarkers for osteoporosis. Sci Rep 8: 8421, 2018.

19. Chen J, Li K, Pang Q, Yang C, Zhang H, Wu F, Cao H, Liu H, Wan Y, Xia W, et al: Identification of suitable reference gene and biomarkers of serum miRNAs for osteoporosis. Sci Rep 6: 36347, 2016. 
20. Ramírez-Salazar EG, Carrillo-Patiño S, Hidalgo-Bravo A Rivera-Paredez B, Quiterio M, Ramírez-Palacios P, Patiño N, Valdés-Flores M, Salmerón J and Velázquez-Cruz R: Serum miRNAs miR-140-3p and miR-23b-3p as potential biomarkers for osteoporosis and osteoporotic fracture in postmenopausal Mexican-Mestizo women. Gene 679: 19-27, 2018.

21. You L, Pan L, Chen L, Gu W and Chen J: miR-27a is essential for the shift from osteogenic differentiation to adipogenic differentiation of mesenchymal stem cells in postmenopausal osteoporosis. Cell Physiol Biochem 39: 253-265, 2016.

22. Wei Q, He M, Chen M, Chen Z, Yang F, Wang H, Zhang J and $\mathrm{He} \mathrm{W}$ : Icariin stimulates osteogenic differentiation of rat bone marrow stromal stem cells by increasing TAZ expression. Biomed Pharmacother 91: 581-589, 2017.

23. Wei Q, Zhang J, Hong G, Chen Z, Deng W, He W and Chen MH: Icariin promotes osteogenic differentiation of rat bone marrow stromal cells by activating the $\mathrm{ER} \alpha-\mathrm{Wnt} / \beta$-catenin signaling pathway. Biomed Pharmacother 84: 931-939, 2016.

24. Wu Y, Xia L, Zhou Y, Xu Y and Jiang X: Icariin induces osteogenic differentiation of bone mesenchymal stem cells in a MAPK-dependent manner. Cell Prolif 48: 375-384, 2015.

25. Liu H, Xiong Y, Zhu X, Gao H, Yin S, Wang J, Chen G, Wang C, Xiang L, Wang $\mathrm{P}$, et al: Icariin improves osteoporosis, inhibits the expression of PPAR $\gamma, \mathrm{C} / \mathrm{EBP} \alpha, \mathrm{FABP} 4 \mathrm{mRNA}, \mathrm{N} 1 \mathrm{ICD}$ and jagged 1 proteins, and increases Notch 2 mRNA in ovariectomized rats. Exp Ther Med 13: 1360-1368, 2017.

26. Wang Z, Wang D, Yang D, Zhen W, Zhang J and Peng S: The effect of icariin on bone metabolism and its potential clinical application. Osteoporos Int 29: 535-544, 2018.

27. Zhang Y, Shen L, Mao Z, Wang N, Wang X, Huang X, Hu Y, Shou D and Wen C: Icariin enhances bone repair in rabbits with bone infection during post-infection treatment and prevents inhibition of osteoblasts by vancomycin. Front Pharmacol 8: 784, 2017.

28. Xu Y, Li L, Tang Y, Yang J, Jin Y and Ma C: Icariin promotes osteogenic differentiation by suppressing Notch signaling. Eur J Pharmacol 865: 172794, 2019.

29. Livak KJ and Schmittgen TD: Analysis of relative gene expression data using real-time quantitative PCR and the 2(-Delta Delta C(T)) method. Methods 25: 402-408, 2001.

30. Kim KM and Lim SK: Role of miRNAs in bone and their potential as therapeutic targets. Curr Opin Pharmacol 16: 133-141, 2014.

31. Ren H, Yu X, Shen G, Zhang Z, Shang Q, Zhao W, Huang J, Yu P, Zhan M, Lu Y, et al: MiRNA-seq analysis of human vertebrae provides insight into the mechanism underlying GIOP. Bone 120 : 371-386, 2019.
32. Xu Q, Cui Y, Luan J, Zhou X, Li H and Han J: Exosomes from $\mathrm{C} 2 \mathrm{C} 12$ myoblasts enhance osteogenic differentiation of MC3T3-E1 pre-osteoblasts by delivering miR-27a-3p. Biochem Biophys Res Commun 498: 32-37, 2018.

33. Ma Y, Shan Z, Ma J, Wang Q, Chu J, Xu P, Qin A and Fan S: Validation of downregulated microRNAs during osteoclast formation and osteoporosis progression. Mol Med Rep 13: 2273-2280, 2016.

34. Guo D, Li Q, Lv Q, Wei Q, Cao S and Gu J: miR-27a targets sFRP1 in hFOB cells to regulate proliferation, apoptosis and differentiation. PLoS One 9: e91354, 2014.

35. Liu Y, Liu C, Zhang A, Yin S, Wang T, Wang Y, Wang M, Liu Y, Ying Q, Sun J, et al: Down-regulation of long non-coding RNA MEG3 suppresses osteogenic differentiation of periodontal ligament stem cells (PDLSCs) through miR-27a-3p/IGF1 axis in periodontitis. Aging (Albany NY) 11: 5334-5350, 2019.

36. Gong Y, Lu J, Yu X and Yu Y: Expression of Sp7 in Satb2-induced osteogenic differentiation of mouse bone marrow stromal cells is regulated by microRNA-27a. Mol Cell Biochem 417: 7-16, 2016.

37. Zhou X, Zhang Z, Feng JQ, Dusevich VM, Sinha K, Zhang H, Darnay BG and de Crombrugghe B: Multiple functions of Osterix are required for bone growth and homeostasis in postnatal mice. Proc Natl Acad Sci USA 107: 12919-12924, 2010.

38. Shi K, Lu J, Zhao Y, Wang L, Li J, Qi B, Li H and Ma C: MicroRNA-214 suppresses osteogenic differentiation of $\mathrm{C} 2 \mathrm{C} 12$ myoblast cells by targeting Osterix. Bone 55: 487-494, 2013.

39. Compston JE, McClung MR and Leslie WD: Osteoporosis. Lancet 393: 364-376, 2019.

40. Guo L, Chen K, Yuan J, Huang P, Xu X, Li C, Qian N, Qi J, Shao Z, Deng L, et al: Estrogen inhibits osteoclasts formation and bone resorption via microRNA-27a targeting PPAR $\gamma$ and APC. J Cell Physiol 234: 581-594, 2018.

41. Gavali S, Gupta MK, Daswani B, Wani MR, Sirdeshmukh R and Khatkhatay MI: Estrogen enhances human osteoblast survival and function via promotion of autophagy. Biochim Biophys Acta Mol Cell Res 1866: 1498-1507, 2019.

42. Anastasilakis AD, Makras P, Pikilidou M, Tournis S, Makris K, Bisbinas I, Tsave O, Yovos JG and Yavropoulou MP: Changes of circulating MicroRNAs in response to treatment with teriparatide or denosumab in postmenopausal osteoporosis. J Clin Endocrinol Metab 103: 1206-1213, 2018.

(i) $($ This work is licensed under a Creative Commons Attribution-NonCommercial-NoDerivatives 4.0 International (CC BY-NC-ND 4.0) License. 